\section{Free-Aspire: A new device for the management of airways clearance in patient with ineffective cough}

\author{
Luca Bertelli, ${ }^{1}$ Giovanni Di Nardo, ${ }^{2}$ \\ Salvatore Cazzato, ${ }^{3}$ Giampaolo Ricci, 1 \\ Andrea Pession 1 \\ 1Department of Pediatrics, University of \\ Bologna, S. Orsola-Malpighi Hospital; \\ 2Pediatric Gastroenterology Unit, AORN \\ Santobono-Pausilipon, Naples: \\ 3Department of Mother and Child \\ Health, Salesi Children's Hospital, \\ Ancona, Italy
}

\begin{abstract}
A 3-year-old girl with Spinal Muscular Atrophy type I presented with a right pneumonia. On physical examination pulmonary auscultation revealed an asymmetry of breath sounds between the 2 hemithoraces, owing to decreased breath sounds in the right hemithorax. Blood tests were normal. The initial working diagnosis was a suspected area of mucus accumulation. A treatment with Free Aspire was started. Within a few days, the girl was discharged with a normal physical examination and X-ray study result. Spinal Muscular Atrophy is a rare neuromuscular disorder characterized by loss of motor neurons and progressive muscle wasting. Cough in these patients result ineffective. Free-Aspire is an electromedical machine for removing bronchoalveolar secretions. The case show that Free Aspire in patients with ineffective cough and impaired removal of secretions is a safe and effective device for the removal of bronchial secretions and could be an another help in the management of airway clearance.
\end{abstract}

\section{Introduction}

The term spinal muscular atrophy (SMA) is applied to a various spectrum of genetic conditions which all affect the spinal motor neuron. The different forms of SMA are associated with different gene mutations and significant phenotypic heterogeneity. SMA is usually categorized by pattern of weakness (i.e. whether predominantly proximal or distal) and mode of inheritance. SMA is a severe neuromuscular condition which primarily involves the lower motor neurons in the spinal cord and brainstem, resulting in progressive muscular atrophy, fasciculation and decreased tendon reflex. Cough in these patients result ineffective. Therefore an important aspect of SMA patients' follow-up is the correct management of respiratory secretion and airways clearance.

\section{Case Report}

A 3-year-old girl with SMA type I was referred to us because of dyspnea. She was the second child of non-consanguineous and healthy parents. The pregnancy was uneventful. She was born after 41 weeks of gestation by normal spontaneous vaginal delivery. Birth weight (3660 g), length (51 $\mathrm{cm})$, and head circumference $(36 \mathrm{~cm})$ were within normal range. There was no family history of neuromuscular disorders, neurologic disorders, or congenital malformations. Both her vital signs and physical examination were normal. During the first months of life the child presented poorfeeding. At 1 months of age neurologic examination of the patient revealed tongue fasciculations, symmetric muscle weakness, generalized hypotonia and areflexia. Laboratory analyses showed normal complete blood counts, liver and kidney function tests and serum electrolytes. Creatine kinase levels were $344 \mathrm{U} / \mathrm{L}$ and serum lactate dehydrogenase levels were $676 \mathrm{U} / \mathrm{L}$. Electromyography presented neurogenic changes, no spontaneous activity, and a markedly reduced innervation pattern more pronounced in distal muscle groups and in the lower extremities. Genetic analysis revealed a homozygous deletion of the SMN1 gene (exon 7). Therefore the diagnosis of SMA type I was made. During the following 3 years of life, the child performed a multidisciplinary disease's specific followup, including chest X-ray, overnight oximetry, gastrointestinal, nutrition and orthopedic evaluations in order to prevent the complications of weakness in childhood spinal muscular atrophy such as respiratory, due to restrictive lung disease; gastrointestinal, in terms of dysphagia and constipation; and orthopedic, with progressive deformities. The child presented dyspnea, therefore she was referred to our pediatric pulmonary clinic by her pediatrician. On physical examination, the child was dyspneic and irritable; pulmonary auscultation revealed an asymmetry of breath sounds between the 2 hemithoraces, owing to decreased breath sounds in the right hemithorax. Oxygen saturation was $94 \%$ to $96 \%$ on room air. Therefore, a chest radiography was immediately performed. The results revealed a right
Correspondence: Luca Bertelli, Clinica Pediatrica, Università degli studi di Bologna, Policlinico S. Orsola-Malpighi, Via Massarenti 11, 40138 Bologna, Italy.

Tel.: +39.051.6364653 - Fax: +39.051.6364441. E-mail: luca.bertelli80@gmail.com

Key words: Spinal Muscular Atrophy; pneumonia; Free Aspire; ineffective cough.

Contributions: LB evaluated the patient and wrote the first draft of the manuscript; GDN evaluated the patient; $\mathrm{SC}$ shared the respiratory management; GR reviewed the manuscript; AP supervised the draft of the manuscript. All authors have seen and approved the final manuscript

Conflict of interest: the authors declare no potential conflict of interest.

Received for publication: 17 June 2017

Accepted for publication: 17 July 2017.

This work is licensed under a Creative Commons Attribution NonCommercial 4.0 License (CC BY-NC 4.0).

CCopyright L. Bertelli et al., 2017

Licensee PAGEPress, Italy

Pediatric Reports 2017; 9:7270

doi:10.4081/pr.2017.7270

pneumonia (Figure 1). Laboratory analyses showed normal complete blood counts, such as liver and kidney function tests, serum electrolytes and C-reactive protein serum value. On the basis of the clinical, radiological and laboratory findings, the initial working diagnosis was a suspected mucus accumulation in the right hemithorax. Therefore, we started a treatment with Free Aspire. The child performed the treatment for 20 minutes three times a day. During the following days both her vital signs and physical examination returned normal. The oxygen saturation was always maintained at greater than $92 \%$, and the objectivity of lung during the following days showed a clear improvement; no asymmetry of breath sounds between the two hemithoraces were found. Within a few days, we repeated chest radiography, and a significant change was found in comparison with the previous result. No right pneumonia was present as shown in Figure 2. Subsequently the girl was discharged with a normal physical examination and X-ray study result.

\section{Discussion}

SMA is an autosomal recessive condi- 
tion characterized by the selective loss of spinal motor neurons, due to lower levels of the survival motor neuron (SMN) protein..$^{1,2}$ SMA is an important genetic cause of infant mortality; nowadays no effective therapies to slow progression of the condition were found. ${ }^{3}$ SMN protein is produced by two genes, SMN1 and SMN2, and in most SMA patients, the former is deleted or nonfunctional. These two genes are different only for a cytosine to thymine transition within exon seven of SMN2. This transition determinates the exclusion of exon seven from $90 \%$ of the SMN2 mRNA transcript and the resulting SMN protein is then quickly eliminated. ${ }^{4}$ Therefore, most of SMN2 mRNA transcripts could not compensate for the loss of SMN1 in SMA patients. 5 SMA is divided into four phenotypes based on the age of onset and motor function reached. Type I has an onset of clinical signs before six months of age, type II between seven and eighteen months, type III between eighteen months and eighteen years, and type IV patients have adult onset. ${ }^{6}$ As in our case, infants affected by SMA type 1 could appear normal prior to developing limb

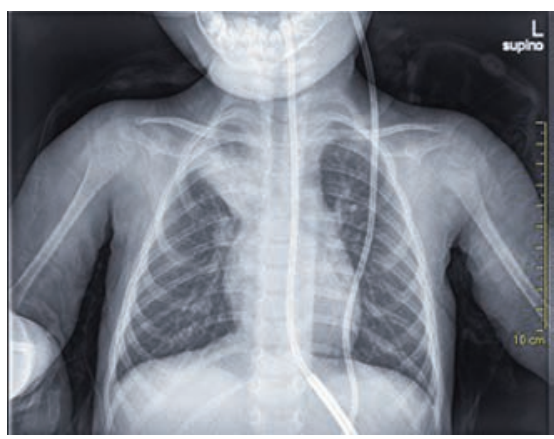

Figure 1. Posteroanterior chest X-ray study performed on admission, showing a right pneumonia.

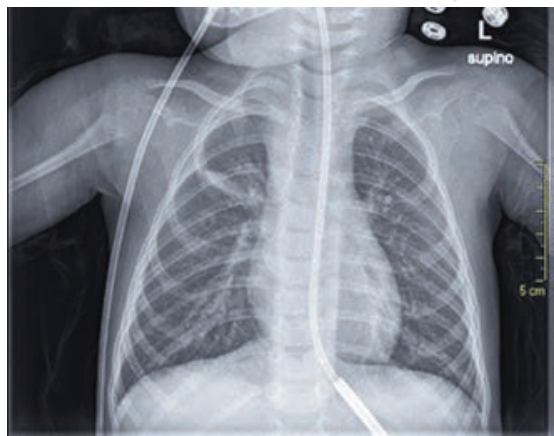

Figure 2. Posteroanterior chest X-ray study returned to normal after Free Aspire treatment. hypotonic weakness, respiratory distress, weak cry and poor feeding. Respiratory features include hypoventilation, weak cough with reduced clearance of lower airway secretions. On the basis of early involvement of the muscles of ventilation, all children with SMA type 1 will present respiratory insufficiency or failure during childhood; 7,8 therefore difficulties with secretion management prior to failure of ventilation could be found. ${ }^{9}$ Ineffective cough and impaired removal of secretions, could favorite recurrent infections of the airways, pneumonia, and atelectasis and these could determinate airways obstruction and lung damage, and further impair the respiratory mechanics. Early strategies is much more satisfactory and effective as compared with treatment during respiratory failure. For airway clearance and management, caregivers should learn to assist coughing when needed, by use of a cough-assist device. Chest physiotherapy and postural drainage could be performed as secretion mobilization techniques. ${ }^{10}$ A new device that could be an help in the airway clearance is FreeAspire (FA). FA (MPR, Medical Products Research Srl, Italy) is an electromedical device for removing broncho-alveolar secretions. The machine utilizes Vaküm technology, which accelerates expiratory flow though the Venturi effect from a special connector. The process happens only during the expiratory phase and is proportional to airflow on spontaneous breathing, according to the natural rhythm of the patient's respiratory function and without needing any patient's collaborations. The secretions slide along the layer of liquid lining the bronchial epithelium until they reach the glottis from where they are swallowed, therefore no respiratory effort is required for the elimination of the secretions. No negative pressure is generated inside the lungs therefore there is no risk of airways collapse. FA does not require an efficient cough and offers a gentle secretion removal solution. Treatments with FA could be performed for $20 \mathrm{~min}$ without determining respiratory fatigue or discomfort. Garuti and colleagues showed how FA for bronchial secretion clearance in patients with limited capacity to collaborate is safe and effective in reducing the impact of respiratory exacerbations in terms of number of PCP visits, days spent in hospital, and days of antibiotic therapy and its regular use maintains this effect in time. 11

\section{Conclusions}

The case shows that FA in patients with ineffective cough and impaired removal of secretions is a safe and effective device for the removal of bronchial secretions and could be an another help in the management of airway clearance.

\section{References}

1. Sleigh JN, Barreiro-Iglesias A, Oliver PL, et al. Chondrolectin affects cell survival and neuronal outgrowth in in vitro and in vivo models of spinal muscular atrophy. Hum Mol Genet 2014;23:85569.

2. Russman BS. Spinal muscular atrophy: clinical classification and disease heterogeneity. J Child Neurol 2007;22: 946-51.

3. Kayadjanian N, Burghes A, Finkel RS, et al. SMA-EUROPE workshop report: Opportunities and challenges in developing clinical trials for spinal muscular atrophy in Europe. Orphanet J Rare Dis 2013;8:44.

4. Porensky PN, Burghes AH. Antisense oligonucleotides for the treatment of spinal muscular atrophy. Hum Gene Ther 2013;24:489-98.

5. Zheleznyakova GY, Kiselev AV, Vakharlovsky VG, et al. Genetic and expression studies of SMN2 gene in Russian patients with spinal muscular atrophy type II and III. BMC Med Genet 2011;12:96.

6. D’Amico A, Mercuri E, Tiziano FD, Bertini E. Spinal muscular atrophy. Orphanet J Rare Dis 2011; 6: 71.

7. Araki S, Hayashi M, Tamagawa K, et al. Neuropathological analysis in spinal muscular atrophy type II. Acta Neuropathol 2003;106:441-8.

8. Kuru S, Sakai M, Konagaya M, et al. An autopsy case of spinal muscular atrophy type III (Kugelberg-Welander disease). Neuropathology 2009;29:637.

9. Simonds AK. Chronic hypoventilation and its management. Eur Respir Rev 2013;22:325-32.

10. Arnold WD, Kassar D, Kissel JT. Spinal muscular atrophy: diagnosis and management in a new therapeutic era. Muscle Nerve 2015;51:157-67.

11. Garuti G, Verucchi E, Fanelli I, et al. Management of bronchial secretions with Free Aspire in children with cerebral palsy: impact on clinical outcomes and healthcare resources. Ital J Pediatr 2016;42:7. 